\title{
Electrophysiological pattern of dream experience
}

\author{
Serena Scarpelli, Luigi De Gennaro \\ Department of Psychology, University of Rome "Sapienza", Rome, Italy \\ Correspondence to: Prof. Luigi De Gennaro. Department of Psychology, University of Rome "Sapienza", Via dei Marsi, 78, 00185 Rome, Italy. \\ Email: luigi.degennaro@uniroma1.it. \\ Provenance: This is a Guest Editorial commissioned by the Editor-in-Chief Baoli Zhu (Jiangsu Provincial Center for Disease Control and Prevention, \\ Nanjing, China). \\ Comment on: Siclari F, Baird B, Perogamvros L, et al. The neural correlates of dreaming. Nat Neurosci 2017;20:872-8.
}

Received: 26 June 2017; Accepted: 27 June 2017; Published: 19 July 2017.

doi: 10.21037/jphe.2017.07.03

View this article at: http://dx.doi.org/10.21037/jphe.2017.07.03

Dreaming is a common human experience investigated from multiple perspectives over the centuries. Recently, this phenomenon has stimulated scientific interest, becoming a peculiar context to study memory processes and consciousness (1).

Since the 1960s it has been clear the possibility of dreaming during all sleep stages, only changing the instruction to collect dream reports (2). Along this line, several studies investigated on the neurobiological bases of dreaming, by mean of neuropsychological, electrophysiological and functional neuroimaging measures. It is necessary to underline that evidence coming from neuroimaging studies are biased by the view that dreaming is an epiphenomenon of rapid eye movement (REM) sleep (3-5). The first (and shared) problem of all these studies is the complexity of this object of research. In fact, it is impossible access directly to dreaming and it is necessary interrupt sleep to collect some information about the mental sleep activity. For this reason, the electrophysiological approach associated to the provoked awakenings represents one of the most preferred method to study dreaming. Clearly, the focus is no longer on dream itself, but on relationship between specific electroencephalographic (EEG) patterns during sleep and dream report upon the awakening. Actually, this is a gold standard approach to study (dream experience DE), and dream recall is the unique viable phenomenon that we can get closer $(6,7)$.

Siclari et al. (8) provided very interesting results on the neural correlates of dreaming. In line with the previous considerations, they confirmed that DE occurs both in REM and non-rapid eye movement (NREM) sleep, performing separated analyses for awakenings upon these different stages. From a methodological point of view, it should be mentioned that they used a within-subjects design and a serial awakenings paradigm during the night, taking under control the intra-individual variability of the EEG pattern. Their main results, consistent in all their experiments, are that DE condition in REM and NREM sleep showed a decrease in low-frequency power (1-4 Hz) over parieto-occipital area, compared to nonexperience (NE). Also high-frequency activity $(25-50 \mathrm{~Hz})$ was related to the DEs. In particular, DE compared to NE was associated to an increase in high-frequencies over the parieto-occipital area in NREM sleep and over the frontal and temporal area in REM sleep (8). As a novel finding, the study identified EEG patterns related to a peculiar kind of dream report-the so-called white dream-corresponding to dream experience without recall (DEWR). This condition was characterized by low-frequency power like in a $\mathrm{DE}$ condition, and no differences in the range of frequency 1-4 $\mathrm{Hz}$ were found between DE and DEWR during NREM sleep. Differently, DEWR compared to NE during REM sleep did not show significant differences. In other words, DE only during NREM sleep is related to slow wave activity (SWA), irrespective to the presence/absence of contents. Instead, significant differences were found for high-frequency EEG activity: DE showed higher highfrequency EEG activity than DEWR in the fronto-parietal regions (8).

Taken together, these results provide evidence that 
dreaming from REM and NREM sleep is related to the same mechanisms and to the same region. This could be considered consistent with the "one-generator" model of dreaming (9). In fact, according to this model, the relationship between dream recall and cortical activation is clearly present in all sleep stages. Convincing evidence supporting this model comes from the research on qualitative differences between REM and NREM dream reports $(10,11)$. In particular, it seems that qualitative features become comparable if the reports are controlled for length (e.g., word count) (10). Furthermore, some studies reported that "dream-like" mental sleep activity is detected also closer to awakening and to sleep onset, in correspondence of higher cortical activity (2). More directly, it should be noted that several studies suggested the relationship between dream recall and cortical arousal (12-14). Recently, it has been observed that also dream recall collected by a nap protocol is associated to a lower delta activity $(0.5-4 \mathrm{~Hz})$ over fronto-temporal and parietal areas during NREM sleep (15).

In general, the pattern of results by Siclari et al. (8) is highly consistent with a one of traditional interpretation regarding neural bases of dream recall: dreaming is linked to a higher cerebral activation as expressed by decreased slow EEG frequencies and increased fast frequencies. In this regard, the authors underlined that the non-recall of sleep mentation upon awakening may be interpreted as absence of consciousness, considering that slow-frequency activity during sleep is associated with neuronal downstates and bistability (16), functionally related to a loss of consciousness (17). However, even if dreaming has been considered as a special context to study consciousness $(1,8,18)$, the notions of DE and consciousness should be cautiously considered equivalent. It should be noted that the assumption that dreaming is generated in the same stage upon which subjects have been awakened is not empirically grounded. We can collect only the dream report, hence we have no way to disentangle whether the dream has actually occurred during a specific period of sleep or not. In other words, we can rule out that SWA may prevent the mental activity at all or whether this kind of activity contributes only to memory consolidation of dream content previously generated. With reference on the issue of "real time prediction" (i.e. the ability to predict the presence/absence of DE on the basis of the EEG activity), Siclari et al. (8) proposed that dream recall could be predicted on the basis of the pattern of activated/deactivated areas as consequence of their experiments. In parts, they strengthen the view of the so-called "brain-reading", previously demonstrated at sleep onset (19).

A very interesting point is the correspondence found between the network of cortical areas associated to DE (and some specific features) during REM sleep and the cortical networks involved in the same mental experience during the wakefulness. For instance, Siclari et al. (8) found that highfrequency activity in the anterior regions (i.e., frontal areas) is associated to thinking dimension of $\mathrm{DE}$, while perceiving dimension correlated with the posterior regions (i.e., parietal, occipital, and temporal areas). Furthermore, dream reports including faces were related to increased highfrequency activity in a cortical region that extends along the fusiform face area. Similarly, the dream reports containing spatial settings were associated with increased highfrequency EEG activity in the right posterior cortex. Also the dream reports containing sense of moving or speech are related, respectively, to the right superior temporal sulcus and the Wernicke's area (8). Despite these areas are involved in the same functions during wakefulness suggesting the "continuity" between the two states of consciousness, the results obtained by Siclari et al. (8) do not address to the question whether these mechanisms are strictly related to the dream generation per se or whether they are an expression of memory consolidation processes, which are necessary to dream recall.

In this regard, some empirical evidence showed a pivotal role of theta $(5-7 \mathrm{~Hz})$ and alpha $(8-12 \mathrm{H})$ bands in dream recall, suggesting that mental sleep activity may be processed as any other declarative mnestic trace (20-22). Bearing in mind the role played by the theta and alpha rhythms in the episodic memory formation and retrieval (23), it has been hypothesized a continuity between the brain mechanisms that modulate episodic memory processes across sleep and wakefulness (21). This is also in line with a psychological perspective, which states that qualitative and quantitative aspects of mental sleep activity are affected by waking-life experiences and feelings $(24,25)$. It has been demonstrated that the frontal theta activity during REM sleep before the awakening predicts a successful recall (21). This pattern was also confirmed by a within-subjects protocol, which found that the frontal theta oscillations in the last segment of REM sleep predict the DR (24). Moreover, several investigations found that 
the alpha EEG activity is involved in the retrieval of sleep mentation. Esposito et al. (20) and Marzano et al. (21) showed that a reduced temporo-parietal alpha during NREM sleep is related to a subsequent DR. All these findings support the idea that the encoding of dream contents during sleep should share some electrophysiological mechanisms with the successful encoding of episodic information during wakefulness. However, it should be mentioned that Siclari et al., (8) recorded EEG during sleep across the $0-50 \mathrm{~Hz}$ frequency range, by restricting their analyses only to two specific frequency ranges, slow $(0-4 \mathrm{~Hz})$ and fast $(20 / 25-50 \mathrm{~Hz})$. Definitely, this complex study represents a crucial upgrade for the present landscape of dream research. However, this choice did not allow to assess the contribution of intermediate EEG frequencies on DE.

\section{Acknowledgements}

Funding: The work was supported by a grant to SS from "Progetti di Avvio alla Ricerca 2016 (AR116154C96089D8), Sapienza, University of Rome".

\section{Footnote}

Conflicts of Interests: The authors have no conflicts of interest to declare.

\section{References}

1. Hobson JA, Pace-Schott EF, Stickgold R. Dreaming and the brain: toward a cognitive neuroscience of conscious states. Behav Brain Sci 2000;23:793-842; discussion 904-1121.

2. Foulkes WD. Dream reports from different stages of sleep. J Abnorm Soc Psychol 1962;65:14-25.

3. Maquet P, Péters J, Aerts J, et al. Functional neuroanatomy of human rapid eye movement sleep and dreaming. Nature 1996;383:163-6.

4. Braun AR, Balkin TJ, Wesensten NJ, et al. Regional Cerebral Blood Flow Throughout the Sleep-Wake Cycle: An H2O-15 Positron Emission Tomography Study. Brain 1997;120:1173-97.

5. Nofzinger EA, Mintun MA, Wiseman M, et al. Forebrain activation in REM sleep: an FDG PET study. Brain Res 1997;770:192-201.

6. De Gennaro L, Marzano C, Cipolli C, et al. How we remember the stuff that dreams are made of: neurobiological approaches to the brain mechanisms of dream recall. Behav Brain Res 2012;226:592-6.

7. Cipolli C, Ferrara M, De Gennaro L, et al. Beyond the neuropsychology of dreaming: Insights into the neural basis of dreaming with new techniques of sleep recording and analysis. Sleep Med Rev 2016. [Epub ahead of print].

8. Siclari F, Baird B, Perogamvros L, et al. The neural correlates of dreaming. Nat Neurosci 2017;20:872-8.

9. Foulkes D. Dreaming: a cognitive-psychological analysis. Hillsdale: Lawrence Erlbaum Associates 1985.

10. Antrobus J. REM and NREM sleep reports: Comparison of word frequencies by cognitive classes. Psychophysiology 1983;20:562-8.

11. Cavallero C, Cicogna P, Natale V, et al. Slow wave sleep dreaming: Dream research. Sleep 1992;15:562-6.

12. Zimmerman WB. Sleep mentation and auditory awakening thresholds. Psychophysiology 1970;6:540-9.

13. Antrobus J. Dreaming: cognitive processes during cortical activation and high afferent thresholds. Psychol Rev 1991;98:96-121.

14. De Gennaro L, Marzano C, Moroni F, et al. Recovery sleep after sleep deprivation almost completely abolishes dream recall. Behav Brain Res 2010;206:293-8.

15. Scarpelli S, D'Atri A, Mangiaruga A, et al. Predicting Dream Recall: EEG Activation During NREM Sleep or Shared Mechanisms with Wakefulness?. Brain Topogr 2017. [Epub ahead of print].

16. Nir Y, Staba RJ, Andrillon T, et al. Regional slow waves and spindles in human sleep. Neuron 2011;70:153-69.

17. Tononi G, Massimini M. Why does consciousness fade in early sleep? Ann N Y Acad Sci 2008;1129:330-4.

18. Siclari F, LaRocque JJ, Bernardi G, et al. The neural correlates of consciousness in sleep: a no-task, within-state paradigm. bioRxiv 2014;012443.

19. Horikawa T, Tamaki M, Miyawaki Y, et al. Neural decoding of visual imagery during sleep. Science 2013;340:639-42.

20. Esposito MJ, Nielsen TA, Paquette T. Reduced alpha power associated with the recall of mentation from Stage 2 and Stage REM sleep. Psychophysiology 2004;41:288-97.

21. Marzano C, Ferrara M, Mauro F, et al. Recalling and forgetting dreams: theta and alpha oscillations during sleep predict subsequent dream recall. J Neurosci 2011;31:6674-83.

22. Scarpelli S, Marzano C, D'Atri A, et al. State-or trait-like individual differences in dream recall: preliminary findings from a within-subjects study of multiple nap REM sleep awakenings. Front Psychol 2015;6:928. 
23. Klimesch W. Memory processes, brain oscillations and EEG synchronization. Int J Psychophysiol 1996;24:61-100.

24. Domhoff GW. The scientific study of dreams: Neural networks, cognitive development, and content analysis.

doi: 10.21037/jphe.2017.07.03

Cite this article as: Scarpelli S, De Gennaro L. Electrophysiological pattern of dream experience. J Public Health Emerg 2017;1:68.
Washington, APA Press, 2003.

25. Schredl M, Hofmann F. Continuity between waking activities and dream activities. Conscious Cogn 2003;12:298-308. 